\title{
A Soft Stairway to Institutions
}

\author{
Antonino Salibra ${ }^{1}$ and Giuseppe Scollo ${ }^{2 \star}$ \\ 1 University of Pisa, Dip. Informatica \\ C.so Italia 40, I-56125 Pisa, Italy \\ 2 University of Twente, Fac. Informatica \\ P.O. Box 217, NL-7500AE Enschede, The Netherlands
}

\begin{abstract}
The notion of institution is dissected into somewhat weaker notions. We introduce a novel notion of institution morphism, and characterize preservation of institution properties by corresponding properties of such morphisms. Target of this work is the stepwise construction of a general framework for translating logics, and algebraic specifications using logical systems. Earlier translations of order-sorted conditional equational logic and of conditional equational logics for partial algebras into equational type logic are revisited in this light. Model-theoretic results relating to compactness are presented as well.
\end{abstract}

\section{Introduction}

In [5] the following question is proposed (among others):

"Anyone who has worked with extensions of $\mathbf{L}_{\omega, \omega}$ knows that some results are entirely "soft" in that they use only very general properties of the logic, properties that carry over to a large number of other logics. Shouldn't such results be part of an axiomatic treatment of logics?"

The meaning of "soft" in the title of this paper is precisely as explained in the question quoted above. Target of our investigation is the notion of institution, which was introduced in [13] as a vehicle for the application of abstract model theory to computer science. The theory of institutions became rapidly popular in computer science, within the algebraic specification community especially, thanks to the neat conceptual support that it offers to modeling of, and reasoning about, a wide variety of specification and programming phenomena. The category-theoretic design of this theory is part of the explanation of that success.

In our case, concrete motivation for the choice of working with institutions was the following, instructive experience. In the elaboration of a few results relating to the expressiveness of equational type logic [18] in comparison to other logics, a number of technical aspects, relating to the translation of the target logic into our framework, show a striking formal commonality over the different translations. These aspects essentially are: representation of models, translation of sentences, structure of completeness proofs. The search for a more general framework, where as much as possible of that commonality could be factored out, is just as natural. For such an

\footnotetext{
* on temporary leave at: LIENS/DMI, École Normale Supérieure, 45 Rue d’Ulm, F-75230 Paris, France
} 
aim, the category of institutions, rooted as it is in abstract model theory, seems to offer the obvious framework to work with. Two facts, however, concur to indicate that this choice is not entirely obvious.

In the first place, we are interested in general tools for lifting results of work done for one logic to other logics, without having to do the work all over again for each of them. In fact, that is the main reason for the popularity of institutions in computer science. However, from the way in which the expressiveness results in [18] are obtained, we are led to observe that pointwise translation of sentences and models is not always that easy to work with. More generally, we wish to translate presentations (i.e. sets of sentences) to presentations, and to associate a class of models in the target logic to each model of the source logic. The notion of institution morphism proposed in [13] deserves generalization, thus.

In the second place, we observed that not every feature of the institution concept had some rôle to play in the trial applications of our interest. The softness of the satisfaction condition, in particular, seems to depend on the scales one is using (more precisely, on the planet where the scales are taken). Although "style has to count for something", in the sense that the temptation toward maximum generality at the expense of intuition should be resisted [5], style and intuition deserve analysis and testing, once in while.

The net result of putting together the two facts that occurred to our observation is thus a strong motivation for a twofold effort towards generality. That is, we wish to dissect the notion of institution into somewhat weaker notions, on the one hand, and to manipulate the resulting weaker structures by means of easier-to-use morphisms, on the other hand. In both directions, of course, we should be able to find the notions considered in [13] as particular cases, when appropriate steps along the specialization 'stairway' are taken. Isolating and characterizing such steps, each independently of the others, meets a principle of orthogonality in the analysis of concepts, appears sensible to intuition, and may yield useful results - for example it might lead one to uncover a wider applicability of known facts.

The sense of, and motivation for (the title of) this paper being now clear, a short outline of the organization of the paper with a summary of results are as follows.

In Sect. 2 we introduce pre-institutions and transformations thereof, and define a few properties that these structures may have, such as satisfaction preservation in pre-institutions, or (full) adequacy and finitarity of pre-institution transformations. The relative expressiveness of pre-institutions is characterized by the existence of suitable transformations. We show how the classical notion of relative expressiveness of logical systems fits in our more general setting.

In Sect. 3 we first show that pre-institutions, with transformations as morphisms, form a category, of which subcategories are obtained by requiring transformation properties-which prove preserved by composition, thus. We also show that preservation of institution properties by transformations is ensured by corresponding properties of those transformations, and that, while every transformation is sound with respect to consequence, adequate transformations are also complete. Invertibility of transformations is then characterized, and sufficient conditions for invertibility are given, which thereby ensure exactly equivalent expressiveness of pre-institutions.

In Sect. 4 a compactness theorem is presented, showing that fully adequate transformations ensure contravariant preservation of compactness, and that consequence- 
compactness (a stronger property) is so preserved if the transformation is also finitary, modulo logical equivalence.

In Sect. 5 a couple of expressiveness results for equational type logic given in [18] are revisited. We show how easily the completeness of the transformations can be proven in the present framework.

In Sect. 6 we quickly review related work; in particular, we indicate how the notion of institution simulation proposed by [4] is related to our notion of preinstitution transformation.

Conclusions are drawn in Sect. 7 , where we also mention a few directions for further work.

\section{Basic Notions and Notations}

A preliminary word about foundations. In this paper we use the term 'set' in a rather comprehensive meaning, that generally includes proper classes. Whenever a need arises to exclude proper classes, we talk of 'small sets'. SET is thus actually a 'metacategory'3, according to [15].

Definition 1. A pre-institution is a 4-tuple $\mathcal{I}=(\operatorname{Sig}, \operatorname{Sen}$, Mod, $\models)$, with:

(i) Sig a category, whose objects are called signatures,

(ii) Sen : Sig $\rightarrow$ SET a functor, sending each signature $\Sigma$ to the set $\operatorname{Sen}(\Sigma)$ of

$\Sigma$-sentences, and each signature morphism $\tau: \Sigma \rightarrow \Sigma^{\prime}$ to the mapping

$\operatorname{Sen}(\tau): \operatorname{Sen}(\Sigma) \rightarrow \operatorname{Sen}\left(\Sigma^{\prime}\right)$ that translates $\Sigma$-sentences to $\Sigma^{\prime}$-sentences,

(iii) Mod : Sig ${ }^{o p} \rightarrow$ SET a functor, sending each signature $\Sigma$ to the set $\operatorname{Mod}(\Sigma)$ of

$\Sigma$-models, and each signature morphism $\tau: \Sigma \rightarrow \Sigma^{\prime}$ to the $\tau$-reduction function $\operatorname{Mod}(\tau): \operatorname{Mod}\left(\Sigma^{\prime}\right) \rightarrow \operatorname{Mod}(\Sigma)$

(iv) $\vDash:|\operatorname{Sig}| \rightarrow\|\operatorname{REL}\| \mid$ a function, ${ }^{4}$ yielding a binary relation $k_{\Sigma} \subseteq \operatorname{Mod}(\Sigma) \times \operatorname{Sen}(\Sigma)$ for each signature $\Sigma$, that is the satisfaction relation between $\Sigma$-models and $\Sigma$-sentences.

Definition 2. Let $\mathcal{I}=(\operatorname{Sig}, \operatorname{Sen}, \operatorname{Mod}, \models)$ be a pre-institution, $\tau: \Sigma \rightarrow \Sigma^{\prime}$ a signature morphism in Sig, $\varphi$ a $\Sigma$-sentence and $\mathrm{M}^{\prime}$ a $\Sigma^{\prime}$-model. With henceforth adoption of the abbreviations:

(a) $\tau \varphi$ for $\operatorname{Sen}(\tau)(\varphi)$,

(b) $\mathbf{M}^{\prime} \tau$ for $\operatorname{Mod}(\tau)\left(\mathbf{M}^{\prime}\right)$,

we say that

(i) reduction preserves satisfaction in $\mathcal{I}$, or that $\mathcal{I}$ has the rps property (or that $\mathcal{I}$ is rps, for short), iff $\mathcal{I}$ meets the following requirement:

$\forall \Sigma, \Sigma^{\prime} \in \operatorname{Sig}, \forall \tau: \Sigma \rightarrow \Sigma^{\prime}, \forall \varphi \in \operatorname{Sen}(\Sigma), \forall M^{\prime} \in \operatorname{Mod}\left(\Sigma^{\prime}\right)$ :

(†) $\mathbf{M}^{\prime} \vDash \Sigma^{\prime} \tau \varphi \Rightarrow \mathbf{M}^{\prime} \tau \vDash_{\Sigma} \varphi$

(ii) expansion preserves satisfaction in $\mathcal{I}$, or that $\mathcal{I}$ has the eps property (or that $\mathcal{I}$ is eps, for short), iff $\mathcal{I}$ meets the following requirement:

$\forall \Sigma, \Sigma^{\prime} \in \operatorname{Sig}, \forall \tau: \Sigma \rightarrow \Sigma^{\prime}, \forall \varphi \in \operatorname{Sen}(\Sigma), \forall M^{\prime} \in \operatorname{Mod}\left(\Sigma^{\prime}\right)$ :

(†) $\mathbf{M}^{\prime} \tau \vDash \Sigma \varphi \Rightarrow \mathrm{M}^{\prime} \vDash \Sigma^{\prime} \tau \varphi$

3 resting at some floor of 'palais Grothendieck'

4 REL is the category of sets with binary relations as morphisms; $\|C\|$ is the set of morphisms of category C. 
(iii) $\mathcal{I}$ preserves satisfaction, or that $\mathcal{I}$ has the ps property (or that $\mathcal{I}$ is ps, for short), iff $\mathcal{I}$ is both rps and eps.

Remark 3. An institution [13] is thus a pre-institution that preserves satisfaction and where model sets and reduction have categorial structure, that is, an institution rather has a functor Mod:Sig ${ }^{o p} \rightarrow \mathrm{CAT}$, sending each signature $\Sigma$ to the category $\operatorname{Mod}(\Sigma)$ of $\Sigma$-models, and each signature morphism $\tau: \Sigma \rightarrow \Sigma^{\prime}$ to the $\tau$-reduction functor $\operatorname{Mod}(\tau): \operatorname{Mod}\left(\Sigma^{\prime}\right) \rightarrow \operatorname{Mod}(\Sigma)$.

It seems interesting to investigate which properties of inctitutions do actually depend on requirements $(t)$ and/or $(\ddagger)$ of Definition 2 , and/or on the categorial structure of model sets and reduction, and which do not, thus holding for larger classes of pre-institutions as well.

According to the motivation proposed in Sect. 1, we are interested in general tools for lifting results from one pre-institution to another pre-institution. Pointwise translation of sentences and models is not always easy to use for this purpose. For example, to recover and possibly further extend the results obtained in [18] we need, more generally, to translate presentations to presentations, and to associate a set of models (in the target pre-institution) to each model of the source pre-institution. A suitable notion of pre-institution morphism will serve to this purpose, for which a few preliminaries are needed.

We recall that the powerset functor $\wp:$ SET $\rightarrow$ SET sends every set to the collection of its subsets ${ }^{5}$, and every function $f: S \rightarrow S^{\prime}$ to the function yielding the f-image of each subset of $S$. The functor $\wp_{+}$is analogously defined, except that the empty set is excluded from the collection $\wp_{+}(S)$, for all sets $S$.

In every pre-institution $\mathcal{I}=($ Sig, Sen, Mod, $\models$ ) we thus define the functor Pre $=$ $\wp$ Sen : Sig $\rightarrow$ SET that sends each signature $\Sigma$ to the set $\operatorname{Pre}(\Sigma)$ of $\Sigma$-presentations, and each signature morphism $\tau: \Sigma \rightarrow \Sigma^{\prime}$ to the mapping $\operatorname{Pre}(\tau): \operatorname{Pre}(\Sigma) \rightarrow \operatorname{Pre}\left(\Sigma^{\prime}\right)$ that translates $\Sigma$-presentations to $\Sigma^{\prime}$-presentations. For convenience, we often write $I=($ Sig, Pre, Mod, $\vDash$ ) instead of the more customary notation introduced in Definition 1 .

Definition4. A pre-institution transformation $\mathcal{T}: \mathcal{I} \rightarrow \mathcal{I}^{\prime}$, where $\mathcal{I}=($ Sig, Pre,Mod, $\models)$ and $\mathcal{I}^{\prime}=\left(\operatorname{Sig}^{\prime}, \operatorname{Pre}^{\prime}\right.$, Mod $\left.^{\prime}, \models^{\prime}\right)$ are pre-institutions, is a 3 -tuple $\mathcal{T}=\left(\mathrm{Si}_{\mathcal{T}}, \operatorname{Pr}_{\mathcal{T}}, \mathrm{Mo}_{\mathcal{T}}\right)$, with:

(i) $\mathrm{Si}_{\mathcal{T}}: \mathrm{Sig} \rightarrow \mathrm{Sig}^{\prime}$ a functor-we shall henceforth write $\Sigma_{\mathcal{T}}$ for $\mathrm{Si}_{\mathcal{T}}(\Sigma)$, and $\tau_{\mathcal{T}}$ for $\mathrm{Si} \tau(\tau)$

(ii) $\operatorname{Pr}_{\mathcal{T}}: \operatorname{Pre} \operatorname{Pre}^{\prime} \circ S_{i}$ a natural transformation, i.e. for each $\Sigma \in$ Sig a function $\operatorname{Pr}_{\mathcal{T}_{\Sigma}}: \operatorname{Pre}(\Sigma) \rightarrow \operatorname{Pre}^{\prime}\left(\Sigma_{\mathcal{T}}\right)$ sending $\Sigma$-presentations to $\Sigma_{\mathcal{T}}$-presentations, such that for every signature morphism $\tau: \Sigma_{1} \rightarrow \Sigma_{2}$ in Sig the following diagram commutes,

\footnotetext{
${ }^{5} \curvearrowleft$ lives in the elevator of 'palais Grothendieck', thus, lifting its argument up one floor.
} 


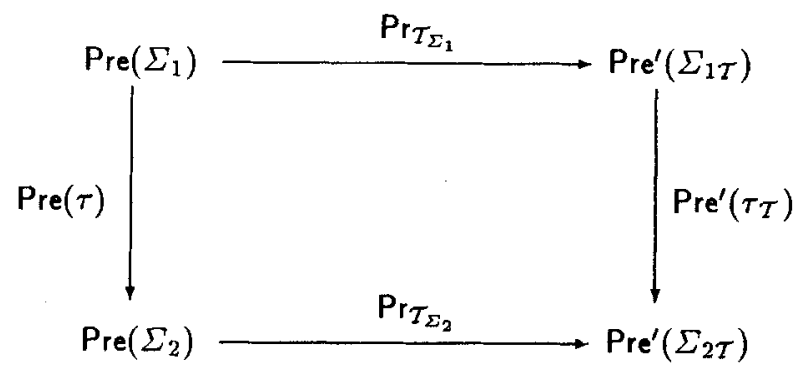

(iii) $\mathrm{M}_{\mathcal{T}}:$ Mod $\rightarrow \wp_{+} \circ \mathrm{Mod}^{\prime} \circ \mathrm{Si}_{\mathcal{T}}$ a natural transformation, i.e. for each $\Sigma \in$ Sig a map $\operatorname{Mo}_{\mathcal{T}_{\Sigma}}: \operatorname{Mod}(\Sigma) \rightarrow \wp_{+}\left(\operatorname{Mod}^{\prime}\left(\Sigma_{\mathcal{T}}\right)\right)$ assigning a nonempty set $\operatorname{Mo\mathcal {T}_{\Sigma }}(\mathrm{M})$ of $\Sigma_{\mathcal{T}^{-}}$ models to each $\Sigma$-model M, such that for every signature morphism $\tau: \Sigma_{1} \rightarrow \Sigma_{2}$ in Sig the following diagram commutes,

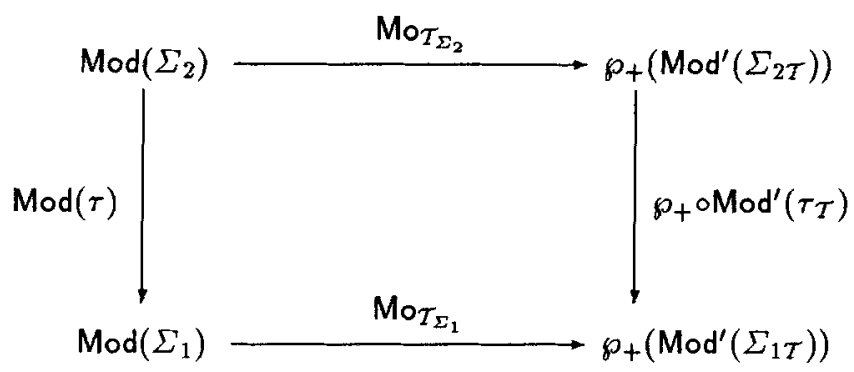

such that the following satisfaction invariant holds:

$$
\forall \Sigma \in \operatorname{Sig}, \forall \mathrm{E} \in \operatorname{Pre}(\Sigma), \forall \operatorname{M} \in \operatorname{Mod}(\Sigma): \quad \mathbf{M} \neq \mathrm{E} \Leftrightarrow \mathbf{M}_{\mathcal{T}} \vDash^{\prime} \mathbf{E}_{\mathcal{T}}
$$

where satisfaction is extended to presentations and model sets in the usual way, and with henceforth adoption of the following abbreviations:

(a) $\mathrm{E}_{\mathcal{T}}$ for $\operatorname{Pr}_{\tau_{\Sigma}}(\mathrm{E})$

(b) $\varphi_{\mathcal{T}}$ for $\{\varphi\}_{\mathcal{T}}$, for a one-sentence presentation $\{\varphi\}$,

(c) $\mathrm{M}_{\mathcal{T}}$ for $\mathrm{Mo}_{\mathcal{T}_{\Sigma}}(\mathrm{M})$,

(d) $\vDash$ for $\models_{\Sigma}$ and $\models^{\prime}$ for $\models^{\prime}{ }_{\Sigma_{T}}$ (and even $\models$ for $\models^{\prime}$, if no ambiguity arises).

The intuitive reason for the non-emptiness requirement on $\mathrm{Mo}_{\mathcal{T}_{\Sigma}}(\mathrm{M})$ is that the existence of a pre-institution transformation is intended to entail the 'representability' of every source model by some target model.

The reason for the requirement expressed by the satisfaction invariant is the soundness of deduction in the image of the transformation with respect to deduction in the source, which fact is apparent from the simple proof of Proposition 16(i).

It may seem strange that presentation transformation is allowed not to respect the set-theoretic structure of presentations, that is, it need not be constructed elementwise. Our design principle, in this case as well as everywhere else in this paper, is that requirements restrict generality, hence there must be sufficient evidence of their necessity to set them a priori rather than introducing them as properties a posteriori. As an instance of the classical "Occam's razor", we have adopted the rule: leges non sunt multiplicanda preter necessitatem. 
Definition 4(iii) doesn't require $\mathbf{M}_{\mathcal{T}}$ to be closed under 'isomorphism'. In fact, no notion of isomorphism between models in a pre-institution is available at all. In some cases, one can replace isomorphism between models by the weaker equivalence that reflects indiscernibility of models by the logical means that is available 'inside the pre-institution', viz. the straightforward generalization of elementary equivalence that is formalized in the first part of the next definition. The second part of the definition formalizes logical equivalence of sentences inside a pre-institution, which fact justifies the overloading of the equivalence symbol.

Definition 5. Let $\mathcal{I}=$ (Sig,Pre,Mod, $\models$ ) be a pre-institution, $\Sigma$ a signature in Sig.

(i) Any two models $M_{1}, M_{2} \in \operatorname{Mod}(\Sigma)$ are $\mathcal{I}$-equivalent, written $M_{1} \equiv{ }_{I} M_{2}$, iff $\forall \mathrm{E} \in \operatorname{Pre}(\Sigma): \mathrm{M}_{1} \vDash \mathrm{E} \Leftrightarrow \mathrm{M}_{2} \vDash \mathrm{E}$.

(ii) Any two sentences $\varphi, \psi \in \operatorname{Sen}(\Sigma)$ are $\mathcal{I}$-equivalent, written $\varphi \equiv_{I} \psi$, iff $\forall \operatorname{M} \in \operatorname{Mod}(\Sigma): M \vDash \varphi \Leftrightarrow M \models \psi$.

Remark 6. In a pre-institution $\mathcal{I}$, two $\Sigma$-sentences are $\mathcal{I}$-equivalent iff no $\Sigma$-model distinguishes them. Conversely, two $\Sigma$-models are $\mathcal{I}$-equivalent iff no $\Sigma$-presentation distinguishes them. The latter notion enables us to introduce a few properties of pre-institution transformations, that we propose without further analysis.

(i) a pre-institution transformation $\mathcal{T}: \mathcal{I} \rightarrow \mathcal{I}^{\prime}$ is insensitive to $\mathcal{I}$-equivalence iff $\mathrm{M}_{1} \equiv_{\mathcal{I}} \mathrm{M}_{2} \Rightarrow \mathrm{M}_{1} \mathcal{\tau}=\mathrm{M}_{2 \mathcal{T}}$

(ii) a pre-institution transformation $\mathcal{T}: \mathcal{I} \rightarrow \mathcal{I}^{\prime}$ is closed under $\mathcal{I}^{\prime}$-equivalence iff $\left(M^{\prime} \in M_{\mathcal{T}} \wedge \mathbf{M}^{\prime \prime} \equiv_{\mathcal{I}^{\prime}} \mathbf{M}^{\prime}\right) \Rightarrow \mathbf{M}^{\prime \prime} \in \mathrm{M}_{\mathcal{T}}$

(iii) a pre-institution transformation $\mathcal{T}: \mathcal{I} \rightarrow \mathcal{I}^{\prime}$ abstracts from indiscernibility iff it is both insensitive to $\mathcal{I}$-equivalence and closed under $\mathcal{I}^{\prime}$-equivalence.

Additional requirements characterize certain classes of pre-institution transformations that will prove useful later.

Definition 7. Let $\mathcal{T}: \mathcal{I} \rightarrow \mathcal{I}^{\prime}$ be a pre-institution transformation, with $\mathcal{I}, \mathcal{I}^{\prime}$ as in Definition 4.

(i) $\mathcal{T}$ is adequate iff it meets the following requirement:

$\forall \Sigma \in \operatorname{Sig}, \forall E \in \operatorname{Pre}(\Sigma), \forall \mathrm{M}^{\prime} \in \operatorname{Mod}^{\prime}\left(\Sigma_{\mathcal{T}}\right)$ :

(§§) $\mathbf{M}^{\prime} \models^{\prime} \mathbf{E}_{\mathcal{T}} \Rightarrow \exists \operatorname{M} \in \operatorname{Mod}(\Sigma): \mathbf{M}^{\prime} \in \mathbf{M}_{\mathcal{T}} \wedge \mathrm{M} \vDash \mathrm{E}$

(ii) $\mathcal{T}$ is fully adequate iff it meets the following requirement:

$\forall \Sigma \in \operatorname{Sig}, \forall \operatorname{M}^{\prime} \in \operatorname{Mod}^{\prime}\left(\Sigma_{\mathcal{T}}\right)$, for all indexed families $\left\{\mathbf{E}_{j}\right\}_{j \in J}$ of $\Sigma$-presentations:

$(\S \S \S) \quad \mathbf{M}^{\prime} \models^{\prime} \bigcup_{j \in J}\left(\mathrm{E}_{j}\right)_{\mathcal{T}} \Rightarrow \exists \operatorname{M} \in \operatorname{Mod}(\Sigma): \mathbf{M}^{\prime} \in \mathbf{M}_{\mathcal{T}} \wedge \mathrm{M} \vDash \bigcup_{j \in J} \mathrm{E}_{j}$.

Although, at a first glance, our definition of adequacy of pre-institution transformations may seem to resemble that of soundness of institution morphisms in [13], such a resemblance is merely formal and has no significance, since model mapping has opposite directions in the two notions. ${ }^{6}$ As we shall see in the next Section, adequacy ensures completeness of the transformation with respect to consequence. Full adequacy is just a stronger form of adequacy (clearly, every fully adequate transformation is adequate as well), that proves connected to compactness of pre-institutions, as shown in Sect. 4.

A sufficient criterion for full adequacy is as follows.

${ }^{6}$ The credit for this clarification is given to Andrzej Tarlecki. 
Lemma 8. Let $\mathcal{T}: \mathcal{I} \rightarrow \mathcal{I}^{\prime}$ be a pre-institution transformation, with $\mathcal{I}, \mathcal{I}^{\prime}$ as in Definition 4. If $\mathcal{T}$ is adequate and meets the following condition: $\forall \Sigma \in \operatorname{Sig}, \forall M_{1}, M_{2} \in \operatorname{Mod}(\Sigma), \forall M^{\prime} \in \operatorname{Mod}^{\prime}\left(\Sigma_{\mathcal{T}}\right):\left(\mathbf{M}^{\prime} \in M_{1 \tau} \wedge M^{\prime} \in M_{2 T}\right) \Rightarrow M_{1}=M_{2 \mathcal{T}}$, then $\mathcal{T}$ is fully adequate.

Proof. Let $\left\{\mathrm{E}_{j}\right\}_{j \in J}$ be a family of $\Sigma$-presentations and $\mathbf{M}^{\prime} \kappa^{\prime} \bigcup_{j \in J}\left(\mathrm{E}_{j}\right)_{\tau}$, thus $\forall j \in J$ : $\mathbf{M}^{\prime} \models^{\prime}\left(\mathbf{E}_{j}\right)_{\mathcal{T}} \cdot \mathcal{T}$ is adequate, so for each $j \in J \exists \mathbf{M}_{j} \in \operatorname{Mod}(\Sigma): \mathbf{M}_{j} \models \mathbf{E}_{j} \wedge \mathbf{M}^{\prime} \in\left(\mathbf{M}_{j}\right)_{\mathcal{T}}$. Then $\left(\mathbf{M}_{j}\right)_{\mathcal{T}} \vDash^{\prime}\left(\mathrm{E}_{j}\right)_{\tau}$ by the satisfaction invariant, and $\forall j, k \in J\left(\mathbf{M}_{j}\right)_{\mathcal{T}}=\left(\mathbf{M}_{k}\right)_{\tau}$ by hypothesis, hence $\left(\mathrm{M}_{k}\right)_{\tau} \vDash F^{\prime}\left(\mathrm{E}_{j}\right)_{\mathcal{T}}$, thus $\mathrm{M}_{k} \vDash \mathrm{E}_{j}$ by the satisfaction invariant. Choose then any $k \in J ; M_{k} \vDash \bigcup_{j \in J} E_{j}$ and $\mathbf{M}^{\prime} \in\left(\mathbf{M}_{k}\right)_{\tau}$ follow at once.

Our notions of adequacy play a significant rôle in the following definition.

Definition 9. Let $\mathcal{I}$ and $\mathcal{I}^{\prime}$ be two pre-institutions.

(i) $\mathcal{I}^{\prime}$ is adequately expressive for $\mathcal{I}$, written $\mathcal{I} \preceq \mathcal{I}^{\prime}$, iff there exists an adequate pre-institution transformation $\mathcal{T}: \mathcal{I} \rightarrow \mathcal{I}^{\prime}$ :

(ii) $\mathcal{I}^{\prime}$ is fully expressive for $\mathcal{I}$, written $\mathcal{I} \sqsubseteq \mathcal{I}^{\prime}$, iff there exists a fully adequate pre-institution transformation $\mathcal{T}: \mathcal{I} \rightarrow \mathcal{I}^{\prime}$.

Fact 10. $\preceq$ and $\sqsubseteq$ are pre-orders.

Definition 11. Let $\mathcal{I}$ and $\mathcal{I}^{\prime}$ be two pre-institutions.

(i) $\mathcal{I}$ and $\mathcal{I}^{\prime}$ have equivalent expressiveness iff $\mathcal{I} \preceq \mathcal{I}^{\prime}$ and $\mathcal{I}^{\prime} \preceq \mathcal{I}$.

(ii) $\mathcal{I}$ and $\mathcal{I}^{\prime}$ have fully equivalent expressiveness iff $\mathcal{I} \sqsubseteq \mathcal{I}^{\prime}$ and $\mathcal{I}^{\prime} \sqsubseteq \mathcal{I}$.

The formal notions of relative expressiveness introduced above generalize the classical notion of relative expressiveness of logical systems in the sense of abstract model theory (see [11]). These have the limitation of being based on first-order models; as a consequence, also the category of (first-order) signatures is fixed for all logical systems. Our notions are more liberal in that only a functor is required between the signature categories, and model-independence is achieved in a most general manner. To clarify this comparison, we show how the classical notion of relative expressiveness between logical systems can be captured by a particular transformation of the corresponding pre-institutions.

Example 12. Let $\mathcal{L}, \mathcal{L}^{\prime}$ be logical systems. According to [11] (p. 194, Definition 1.2 ), and [10] (p. 27, Definition 1.1.1), $\mathcal{L}^{\prime}$ is at least as strong as $\mathcal{L}$, which is written $\mathcal{L} \leq \mathcal{L}^{\prime}$, iff for every first-order signature $\Sigma$, for every $\Sigma$-sentence $\varphi$ in $\mathcal{L}$ there is some $\Sigma$-sentence $\psi$ in $\mathcal{L}^{\prime}$ that has the same models. Let $\mathcal{L}_{I}, \mathcal{L}_{I}^{\prime}$ be the pre-institutions that respectively correspond to $\mathcal{L}, \mathcal{L}^{\prime}$, with $\mathrm{Sig}=\mathrm{Sig}^{\prime}$ the category of first-order signatures having only renamings ${ }^{7}$ as morphisms. A transformation $\mathcal{T}: \mathcal{L}_{I} \rightarrow \mathcal{L}_{I}^{\prime}$ which captures the classical notion of relative expressiveness mentioned above is as follows: whenever $\mathcal{L} \leq \mathcal{L}^{\prime}$, define $\Sigma_{\mathcal{T}}=\Sigma, \mathrm{E}_{\mathcal{T}}=\left\{\psi \mid \exists \varphi \in \mathrm{E}: \operatorname{Mod}(\varphi)=\operatorname{Mod}^{\prime}(\psi)\right\}, \mathrm{M}_{\mathcal{T}}=\{\mathrm{M}\}$. It is easy to see (using Lemma 8 ) that $\mathcal{T}$ is a fully adequate transformation.

7 that is, bijective arity-preserving maps 


\section{Basic Facts}

The extent to which the properties introduced in Definition 2 for pre-institutions, and in Definition 7 for pre-institution transformations, are (not) relevant is revealed by the following facts.

Proposition 13. Let $\mathcal{T}: \mathcal{I} \rightarrow \mathcal{I}^{\prime}$ be a pre-institution transformation, with $\mathcal{I}$, $\mathcal{I}^{\prime}$ as in Definition 4 , and $\Sigma \in$ Sig.

(i) If $\mathbf{M}^{\prime} \in \mathbf{M}_{\mathcal{T}}$ for some $\mathbf{M}$, then $\mathbf{M}^{\prime} \models^{\prime} \emptyset_{\mathcal{T}}$ (where $\emptyset$ is the empty $\Sigma$-presentation).

(ii) Let $R \subseteq \operatorname{Mod}(\Sigma)$ and $\varphi \in \operatorname{Sen}(\Sigma)$. Then $R \vDash \varphi \Leftrightarrow \bigcup_{M \in R} M_{\mathcal{T}} \vDash^{\prime} \varphi_{\mathcal{T}}$.

(iii) Let $\operatorname{M\in Mod}(\Sigma)$ and $\operatorname{E} \in \operatorname{Pre}(\Sigma)$. Then $\mathbf{M}_{\mathcal{T}} \vDash^{\prime} \mathrm{E}_{\mathcal{T}} \Leftrightarrow \mathbf{M}_{\mathcal{T}} \vDash^{\prime} \bigcup_{\varphi \in E^{\varphi}}$.

Proof.

(i) and (ii) are immediate consequence of the satisfaction invariant (Definition 4).

(iii) If $\mathrm{M}_{\mathcal{T}} \vDash^{\prime} \mathrm{E}_{\mathcal{T}}$, then $\mathrm{M} \vDash \mathrm{E}$ by the satisfaction invariant, that is $\mathrm{M} \vDash \varphi$ for all $\varphi \in \mathrm{E}$, which entails $\mathrm{M}_{\mathcal{T}} \vDash \vDash^{\prime} \varphi_{\mathcal{T}}$ again by the satisfaction invariant.

Conversely, if $\forall \varphi \in \mathrm{E} \mathrm{M}_{\mathcal{T}} \vDash \models^{\prime} \varphi_{\mathcal{T}}$, then $\forall \varphi \in \mathrm{E} \mathrm{M} \vDash \varphi$ by the satisfaction invariant, therefore $\mathrm{M} \vDash \mathrm{E}$, whence $\mathrm{M}_{\mathcal{T}} \vDash^{\prime} \mathrm{E}_{\mathcal{T}}$ again by the satisfaction invariant.

We introduced pre-institution transformations as "morphisms", but we did not fully justify that terminology as yet. The following proposition deals with this kind of details.

\section{Proposition 14. (pre-institution categories)}

(i) The identity transformation $\mathcal{E}_{I}: \mathcal{I} \rightarrow \mathcal{I}$ (where $\Sigma_{\mathcal{E}_{I}}=\Sigma, \mathrm{E}_{\mathcal{E}_{I}}=\mathrm{E}, \mathrm{M}_{\mathcal{E}_{I}}=\{\mathrm{M}\}$ ) meets the satisfaction invariant, and is fully adequate.

(ii) If $\mathcal{T}: \mathcal{I} \rightarrow \mathcal{I}^{\prime}$ and $\mathcal{T}^{\prime}: \mathcal{I}^{\prime} \rightarrow \mathcal{I}^{\prime \prime}$ are pre-institution transformations, then so is their composition $\mathcal{T}^{\prime} \circ \mathcal{T}: \mathcal{I} \rightarrow \mathcal{I}^{\prime \prime}$, where:

$\Sigma_{T^{\prime} \circ T}=\left(\Sigma_{T}\right)_{T^{\prime}}$

$\tau_{T^{\prime} \circ T}=\left(\tau_{T}\right)_{T^{\prime}}$

$\mathrm{E}_{T^{\prime} \circ T}=\left(\mathrm{E}_{T}\right)_{T^{\prime}}$

$\mathrm{M}_{\mathcal{T}^{\prime} \circ T}=\bigcup_{M^{\prime} \in M_{\mathcal{T}}} \mathbf{M}^{\prime} \mathcal{T}^{\prime}$.

(iii) $\mathcal{T}^{\prime} \circ \mathcal{T}$ is adequate if both $\mathcal{T}$ and $\mathcal{T}^{\prime}$ are adequate.

(iv) $\mathcal{T}^{\prime} \circ \mathcal{T}$ is fully adequate if both $\mathcal{T}$ and $\mathcal{T}^{\prime}$ are fully adequate.

(v) Pre-institutions, together with transformations as morphisms form a category PT, of which a subcategory APT is obtained by taking only adequate transformations as morphisms, of which a subcategory FAPT is obtained by taking only fully adequate transformations as morphisms.

Proof.

(i) Immediate, using Lemma 8.

(ii) We have only to check that $\mathcal{T}^{\prime} \circ \mathcal{T}$ meets the satisfaction invariant, which easily follows from the definition of $\mathcal{T}^{\prime} \circ \mathcal{T}$.

(iii) If $\mathbf{M}^{\prime \prime} \vDash \mathbf{E}_{\mathcal{T}}$ oT then, since $\mathcal{T}^{\prime}$ is adequate, there exists $M^{\prime} \in \operatorname{Mod}\left(\Sigma_{\mathcal{T}}\right)$ such that $M^{\prime \prime} \in M^{\prime} \mathcal{T}^{\prime}$ and $M^{\prime} \vDash E_{\mathcal{T}}$. This, and the adequacy of $\mathcal{T}$ entail that there exists $M \in \operatorname{Mod}(\Sigma)$ such that $M^{\prime} \in M_{\mathcal{T}}$ and $M \vDash E$. Then $M^{\prime \prime} \in M^{\prime}{ }^{\prime}$ and $M^{\prime} \in M_{\mathcal{T}}$ together imply $\mathrm{M}^{\prime \prime} \in \mathrm{M}_{\mathcal{T}}{ }^{\circ} \circ \mathrm{T}$, and since $\mathrm{M} \vDash \mathrm{E}$, it follows that $\mathcal{T}^{\prime} \circ \mathcal{T}$ is adequate as well. 
(iv) If $\mathbf{M}^{\prime \prime} \models\left(\mathrm{E}_{j}\right)_{\mathcal{T}} \circ \mathrm{T}$ for all $j \in J$, then the full adequacy of $\mathcal{T}^{\prime}$ entails the existence of a $\mathbf{M}^{\prime} \in \operatorname{Mod}\left(\Sigma_{\mathcal{T}}\right)$ such that $\mathbf{M}^{\prime \prime} \in \mathbf{M}^{\prime} \mathcal{T}^{\prime}$ and $\mathbf{M}^{\prime} \vDash\left(\mathbf{E}_{j}\right)_{\mathcal{T}}$ for all $j \in J$. The full adequacy of $\mathcal{T}$ then entails that there exists $\operatorname{M} \in \operatorname{Mod}(\Sigma)$ such that $\mathbf{M}^{\prime} \in \mathrm{M}_{\mathcal{T}}$ and $\mathbf{M} \models \mathrm{E}_{j}$ for all $j \in J$. Then $\mathbf{M}^{\prime \prime} \in \mathrm{M}^{\prime}{ }_{\mathcal{T}}$, and $\mathbf{M}^{\prime} \in \mathbf{M}_{\mathcal{T}}$ together imply $\mathrm{M}^{\prime \prime} \in \bigcup_{M^{\prime} \in M_{T}} \mathrm{M}^{\prime} \mathcal{T}^{\prime}=\mathrm{M}_{\mathcal{T}} \circ T$, and since $\mathrm{M} \vDash \mathrm{E}_{j}$ for all $j \in J$, it follows that $\mathcal{T}^{\prime} \circ \mathcal{T}$ is fully adequate as well.

(v) Validity of the standard categorial laws in PT is easily verified. APT is a subcategory of PT since identities are adequate and composition preserves adequacy. FAPT is a subcategory of APT since identities are fully adequate and composition preserves full adequacy.

Note that the identity transformation of Proposition 14(i) enjoys none of the properties introduced in Remark 6. Clearly, this is the 'safest' identity, in that coarser identities can always be obtained by appropriate quotients. For example, the coarser identity transformation $\mathcal{A}_{I}: \mathcal{I} \rightarrow \mathcal{I}$, where $\Sigma_{\mathcal{A}_{I}}=\Sigma, \mathbf{E}_{\mathcal{A}_{I}}=\mathbf{E}, \mathbf{M}_{\mathcal{A}_{I}}=\left\{\mathbf{M}^{\prime} \mid \mathbf{M}^{\prime} \equiv_{\mathcal{I}} \mathbf{M}\right\}$, abstracts from indiscernibility (proof: $\mathcal{A}_{I}$ is eyidently closed under $\mathcal{I}$-equivalence; its insensitivity to $\mathcal{I}$-equivalence follows from extensionality of sets).

The following fact shows that pre-institution transformations enjoy 'contravariant' preservation of the rps and eps properties. This fact seems to be only the first phenomenon of a wealthy situation; the compactness theorem in the next Section is another such case. Preservation is 'contravariant' in the sense that, if $\mathcal{T}: \mathcal{I} \rightarrow \mathcal{I}^{\prime}$ is a pre-institution transformation and $\mathcal{I}^{\prime}$ has the property under consideration, then $\mathcal{I}$ has that property as well.

These results demonstrate the usefulness of our notion of transformation, in that they support interesting proof techniques. For example, if a proof of a certain theorem in a pre-institution $\mathcal{I}$ is sought, and the theorem is known to hold in a pre-institution $\mathcal{I}^{\prime}$, it will suffice to find a transformation $\mathcal{T}: \mathcal{I} \rightarrow \mathcal{I}^{\prime}$, since this allows the transfer of the known result back to $\mathcal{I}$.

Another, perhaps more interesting application of these results is concerned with negative results on comparing the expressiveness of pre-institutions, in the sense of Definition 9. The proof technique, which has a 'contrapositive' flavour, simply consists in showing that some of the properties which are contravariantly preserved by (possibly 'suitable') pre-institution transformations is enjoyed by $\mathcal{I}^{\prime}$ but not by $\mathcal{I}$. In such a case, then, one can infer that no ('suitable') pre-institution transformation $\mathcal{T}: \mathcal{I} \rightarrow \mathcal{I}^{\prime}$ exists (where 'suitable' means: with some additional property, such as adequacy). An application of this proof method is in Sect. 5.1 below.

Proposition 15. Let $\mathcal{T}: \mathcal{I} \rightarrow \mathcal{I}^{\prime}$ be a pre-institution transformation, with $\mathcal{I}, \mathcal{I}^{\prime}$ as in Definition 4.

(i) If $\mathcal{I}^{\prime}$ is rps, then $\mathcal{I}$ is rps.

(ii) If $\mathcal{I}^{\prime}$ is eps, then $\mathcal{I}$ is eps.

(iii) If $\mathcal{I}^{\prime}$ is ps, then $\mathcal{I}$ is ps.

Proof. For all signature morphisms $\tau: \Sigma_{2} \rightarrow \Sigma_{1}$ in $\operatorname{Sig}, \forall \operatorname{M\in Mod}\left(\Sigma_{2}\right), \forall \varphi \in \operatorname{Sen}\left(\Sigma_{1}\right)$ :

(i) If $\mathbf{M} \vDash \tau \varphi$, then $\forall \mathbf{M}^{\prime} \in \mathrm{M}_{\mathcal{T}}: \mathbf{M}^{\prime} \models^{\prime}(\tau \varphi)_{\mathcal{T}}$ by the satisfaction invariant, therefore $\forall \mathbf{M}^{\prime} \in \mathbf{M}_{\mathcal{T}}: \mathbf{M}^{\prime} \tau_{\mathcal{T}} \vDash \vDash^{\prime} \varphi_{\mathcal{T}}$ by hypothesis and since $(\tau \varphi)_{\mathcal{T}}=\tau_{\mathcal{T}} \varphi_{\mathcal{T}}$ according to the commutativity of the diagram in Definition 4(ii). Moreover, the commutativity of 
the diagram in Definition 4(iii) entails that $(\mathrm{M} \tau)_{\tau}=\left\{\mathrm{M}^{\prime} \tau_{\mathcal{\tau}} \mid \mathrm{M}^{\prime} \in \mathrm{M}_{\tau}\right\}$, therefore $(\mathrm{M} \tau)_{\mathcal{T}} \vDash^{\prime} \varphi_{\mathcal{T}}$, whence $\mathrm{M} \tau \vDash \varphi \varphi$ by the satisfaction invariant.

(ii) If $\mathrm{M} \tau \models \varphi$, then $(\mathrm{M} \tau)_{\tau} \vDash^{\prime} \varphi_{\mathcal{T}}$ by the satisfaction invariant, therefore $\forall \mathrm{M}^{\prime} \in \mathrm{M}_{\tau}$ : $\mathrm{M}^{\prime} \tau_{\mathcal{T}} \models^{\prime} \varphi_{\mathcal{T}}$ since the commutativity of the diagram in Definition 4(ii) entails that $(\mathbf{M} \tau)_{\mathcal{T}}=\left\{\mathbf{M}^{\prime} \tau_{\tau} \mid \mathbf{M}^{\prime} \in \mathrm{M}_{\mathcal{T}}\right\}$. Then $\forall \mathbf{M}^{\prime} \in \mathrm{M}_{\tau}: \mathbf{M}^{\prime} \models^{\prime}(\tau \varphi)_{\mathcal{T}}$ holds by hypothesis and since $(\tau \varphi)_{\tau}=\tau_{\tau} \varphi_{\mathcal{T}}$ according to the commutativity of the diagram in Definition 4 (ii), therefore $\mathrm{M} \models \tau \varphi$ by the satisfaction invariant.

(iii) From (i) and (ii), according to Definition 2(iii).

The reason why (full) adequacy of the transformation is required as a criterion for (full) expressiveness is apparent from the following fact, where $\vDash$ denotes logical consequence, defined in the usual semantical way.

Proposition 16. Let $\mathcal{T}: \mathcal{I} \rightarrow \mathcal{I}^{\prime}$ be a pre-institution transformation, with $\mathcal{I}, \mathcal{I}^{\prime}$ as in Definition 4. Then $\forall \varphi \in \operatorname{Sen}(\Sigma), \forall \psi \in \operatorname{Sen}^{\prime}\left(\Sigma_{\mathcal{T}}\right), \forall \mathrm{E}, \mathrm{E}_{j} \in \operatorname{Pre}(\Sigma)$ :

(i) $\mathrm{E}_{T} \vDash \varphi_{T} \Rightarrow \mathrm{E} \vDash \varphi$.

(ii) $\mathrm{E} \models \varphi \Rightarrow \mathrm{E}_{\mathcal{T}} \models \varphi_{\mathcal{T}}$ if $\mathcal{T}$ is adequate.

(iii) $\bigcup_{j \in J}\left(\mathrm{E}_{j}\right)_{\mathcal{T}} \vDash \psi \Rightarrow\left(\bigcup_{j \in J} \mathrm{E}_{j}\right)_{\tau} \vDash \psi$ if $\mathcal{T}$ is adequate.

(iv) $\left(\bigcup_{j \in J} \mathrm{E}_{j}\right)_{\tau} \vDash \psi \Rightarrow \bigcup_{j \in J}\left(\mathrm{E}_{j}\right)_{\tau} \vDash \psi$ if $\mathcal{T}$ is fully adequate.

Proof.

(i) Let $\mathrm{M} \in \operatorname{Mod}(\Sigma)$, assume $\mathrm{M} \vDash \mathrm{E}$. By the satisfaction invariant $\mathrm{M}_{\mathcal{T}} \vDash \mathrm{E}_{\mathcal{T}}$, therefore $\mathrm{M}_{\mathcal{T}} \vDash \varphi_{\mathcal{T}}$ by hypothesis, whence $\mathrm{M} \vDash \varphi$ by the satisfaction invariant.

(ii) Let $\mathrm{M}^{\prime} \in \operatorname{Mod}^{\prime}\left(\Sigma_{\mathcal{T}}\right)$, assume $\mathrm{M}^{\prime} \vDash \mathrm{E}_{\mathcal{T}}$. By adequacy of $\mathcal{T}$, there exists $\operatorname{M} \in \operatorname{Mod}(\Sigma)$ such that $M^{\prime} \in M_{\mathcal{T}}$ and $M \vDash E$, hence $M \models \varphi$ by hypothesis, whence $M^{\prime} \vDash \varphi_{\mathcal{T}}$ by the satisfaction invariant.

(iii) Let $\mathbf{M}^{\prime} \in \operatorname{Mod}^{\prime}\left(\Sigma_{T}\right)$, assume $\mathbf{M}^{\prime} \vDash\left(\bigcup_{j \in J} \mathbf{E}_{j}\right)_{\tau}$. Then there exists $\operatorname{M\in Mod}(\Sigma)$ such that $\mathbf{M}^{\prime} \in \mathrm{M}_{\mathcal{T}}$ and $\mathrm{M} \models \bigcup_{j \in J} \mathrm{E}_{j}$, by adequacy of $\mathcal{T}$. Thus $\forall j \in J: M \vDash \mathrm{E}_{j}$, hence $\forall j \in J: \mathbf{M}_{\mathcal{T}} \vDash\left(\mathrm{E}_{j}\right)_{\mathcal{T}}$ by the satisfaction invariant, so $\mathbf{M}_{\mathcal{T}} \vDash \bigcup_{j \in J}\left(\mathrm{E}_{j}\right)_{\mathcal{T}}$, whence $\mathbf{M}_{\mathcal{T}} \vDash \psi$ by hypothesis. Since $\mathbf{M}^{\prime} \in \mathbf{M}_{\mathcal{T}}, \mathbf{M}^{\prime} \vDash \psi$ follows.

(iv) Let $\mathbf{M}^{\prime} \in \operatorname{Mod}^{\prime}\left(\Sigma_{\mathcal{T}}\right)$, assume $\mathbf{M}^{\prime} \models \bigcup_{j \in J}\left(\mathbf{E}_{j}\right)_{\tau}$. Since $\mathcal{T}$ is fully adequate, there exists $M \in \operatorname{Mod}(\Sigma)$ such that $\mathbf{M}^{\prime} \in \mathbf{M}_{\mathcal{T}}$ and $\mathbf{M} \vDash \bigcup_{j \in J} \mathbf{E}_{j}$, hence $\mathbf{M}_{\mathcal{T}} \vDash\left(\bigcup_{j \in J} \mathrm{E}_{j}\right)_{\mathcal{T}}$ by the satisfaction invariant, whence $\mathbf{M}_{\mathcal{T}} \vDash \psi$ by hypothesis. Since $\mathbf{M}^{\prime} \in \mathbf{M}_{\mathcal{T}}$, it follows that $\mathrm{M}^{\prime} \vDash \psi$.

If two pre-institutions enjoy equivalent expressiveness, it is sensible to wonder whether the transformations that establish the equivalence are 'inverse' to each other in some sense. The first such sense which comes to mind is the categorial one, viz. that of pre-institution isomorphism, which requires the composition of the two preinstitution transformations, in either order, to be the identity transformation on the corresponding pre-institution. Clearly, the isomorphism condition is stronger than that required for equivalent expressiveness, and is veritably too strong for practical purposes-based as it is on the smallest identity at all levels: signatures, presentations, models.

The strength introduced by the isomorphism condition can be weakened in various ways, e.g. according to coarser equivalences on presentations or on models. Among such possibilities, we formalize a notion of equivalence that requires the transformation of logical theories to be the identity; more precisely, the presentation 
obtained by applying such a transformation and then its inverse to any given presentation is required to have exactly the same consequences as the original presentation. As usual, if $\mathrm{E}$ is a $\Sigma$-presentation, $\mathrm{Th}(\mathrm{E})$ denotes the closure of $\mathrm{E}$ under consequence, whereas if $\mathbf{M}$ is a $\Sigma$-model, then $\mathrm{Th}(\mathrm{M})$ denotes the largest $\Sigma$-presentation that is satisfied by $M$.

Definition 17. Let $\mathcal{T}: \mathcal{I} \rightarrow \mathcal{I}^{\prime}$ be a pre-institution transformation, with $\mathcal{I}, \mathcal{I}^{\prime}$ as in Definition $4 . \mathcal{T}$ is invertible if there exists a pre-institution transformation $\mathcal{R}: \mathcal{I}^{\prime} \rightarrow \mathcal{I}$ such that $\forall \Sigma \in \operatorname{Sig}, \forall \mathrm{E} \in \operatorname{Pre}(\Sigma)$ : $\operatorname{Th}(\mathrm{E})=\operatorname{Th}\left(\left(\mathrm{E}_{\mathcal{T}}\right)_{\mathcal{R}}\right)$, in which case $\mathcal{R}$ is termed inverse of $\mathcal{T}$, and the two pre-institutions $\mathcal{I}, \mathcal{I}^{\prime}$ have exactly equivalent expressiveness.

As a simple illustration, with reference to Example 12, it is easily seen that if $\mathcal{L} \leq \mathcal{L}^{\prime}$ and $\mathcal{L}^{\prime} \leq \mathcal{L}$, then the transformation $\mathcal{T}: \mathcal{L}_{I} \rightarrow \mathcal{L}_{I}^{\prime}$ is invertible (indeed it has a fully adequate inverse), i.e. $\mathcal{L}_{I}$ and $\mathcal{L}_{I}^{\prime}$ have exactly equivalent expressiveness.

The Galois connection nature of invertible pre-institution transformations is revealed by the following characterization.

Proposition 18. Let $\mathcal{T}: \mathcal{I} \rightarrow \mathcal{I}^{\prime}$ and $\mathcal{R}: \mathcal{I}^{\prime} \rightarrow \mathcal{I}$ be two pre-institution transformations, with $\mathcal{I}, \mathcal{I}^{\prime}$ as in Definition 4 . The following conditions are equivalent:

(a) $\mathcal{R}$ is inverse of $\mathcal{T}$

(b) $\mathcal{T}$ is inverse of $\mathcal{R}$

(c) $\forall \Sigma \in \operatorname{Sig}, \forall \mathrm{E} \in \operatorname{Pre}(\Sigma), \forall \mathrm{M}^{\prime} \in \operatorname{Mod}\left(\Sigma_{\mathcal{T}}\right): \mathrm{M}^{\prime} \vDash^{\prime} \mathrm{E}_{\mathcal{T}} \Leftrightarrow\left(\mathrm{M}^{\prime}\right)_{\mathcal{R}} \vDash \mathrm{E}$

(d) $\forall \Sigma^{\prime} \in \operatorname{Sig}^{\prime}, \forall \mathrm{E}^{\prime} \in \operatorname{Pre}^{\prime}\left(\Sigma^{\prime}\right), \forall \operatorname{M} \in \operatorname{Mod}\left(\Sigma_{\mathcal{R}}^{\prime}\right): \mathbf{M} \vDash \mathbf{E}_{\mathcal{R}}^{\prime} \Leftrightarrow \mathbf{M}_{\mathcal{T}} \vDash F^{\prime} \mathbf{E}^{\prime}$

Proof. It is sufficient to show the two implications (a) $\Rightarrow(c),(d) \Rightarrow(a)$, since the two implications $(b) \Rightarrow(d),(c) \Rightarrow(b)$, respectively follow by symmetry.

(a) $\Rightarrow$ (c):

Let $\mathrm{E}$ be a $\Sigma$-presentation and $\mathrm{M}^{\prime}$ a $\Sigma_{\mathcal{T}}$-model. Then $\mathrm{M}^{\prime} \vDash^{\prime} \mathrm{E}_{\mathcal{T}} \Leftrightarrow\left(\mathrm{M}^{\prime}\right)_{\mathcal{R}} \vDash\left(\mathrm{E}_{\mathcal{T}}\right)_{\mathcal{R}}$ by the satisfaction invariant, and $\left(\mathrm{M}^{\prime}\right)_{\mathcal{R}} \vDash\left(\mathrm{E}_{\mathcal{T}}\right)_{\mathcal{R}} \Leftrightarrow\left(\mathrm{M}^{\prime}\right)_{\mathcal{R}} \vDash \mathrm{E}$ by hypothesis (a). (d) $\Rightarrow$ (a):

Let $\mathrm{E}$ be a $\Sigma$-presentation and $\mathrm{M}$ a $\Sigma$-model. Then $\mathrm{M} \vDash \mathrm{E} \Leftrightarrow \mathrm{M}_{\mathcal{T}} \models^{\prime} \mathrm{E}_{\mathcal{T}}$ by the satisfaction invariant, and $\mathrm{M}_{\mathcal{T}} \models^{\prime} \mathrm{E}_{\mathcal{T}} \Leftrightarrow \mathrm{M} \vDash\left(\mathrm{E}_{\mathcal{T}}\right)_{\mathcal{R}}$ by hypothesis (d).

Sufficient conditions for exactly equivalent expressiveness may be of help in the construction of such equivalences. Of the two conditions given below, the second one is stronger, but may turn out to be more useful in practice.

Proposition 19. Let $\mathcal{T}: \mathcal{I} \rightarrow \mathcal{I}^{\prime}$ and $\mathcal{R}: \mathcal{I}^{\prime} \rightarrow \mathcal{I}$ be two pre-institution transformations, with $\mathcal{I}, \mathcal{I}^{\prime}$ as in Definition 4 . The following conditions are sufficient for $\mathcal{R}$ to be inverse of $\mathcal{T}$.

(i) $\forall \Sigma \in \operatorname{Sig}, \forall \operatorname{M} \in \operatorname{Mod}(\Sigma), \forall E^{\prime} \in \operatorname{Pre}\left(\Sigma_{\mathcal{T}}\right): \operatorname{M\in }\left(\mathbf{M}_{\mathcal{T}}\right)_{\mathcal{R}} \wedge\left(\mathrm{M} \vDash \mathrm{E}_{\mathcal{R}}^{\prime} \Rightarrow \mathbf{M}_{\mathcal{T}} \vDash F^{\prime} E^{\prime}\right)$

(ii) $\forall \Sigma \in \operatorname{Sig}, \forall \operatorname{M} \in \operatorname{Mod}(\Sigma): \operatorname{M} \in\left(M_{\tau}\right)_{\mathcal{R}} \wedge\left(M_{\mathcal{T}}\right)_{\mathcal{R}} \subseteq \operatorname{Mod}(\operatorname{Th}(\mathbf{M}))$

Proof.

(i) Let $M$ be a $\Sigma$-model and $E$ a $\Sigma$-presentation. If $M \models E$, then $\left(\mathrm{M}_{\mathcal{T}}\right)_{\mathcal{R}} \vDash\left(\mathrm{E}_{\mathcal{T}}\right)_{\mathcal{R}}$ by the satisfaction invariant, hence $M \models\left(E_{T}\right)_{\mathcal{R}}$ by the hypothesis $M \in\left(M_{\mathcal{T}}\right)_{\mathcal{R}}$. On the other hand, $M \vDash\left(E_{\mathcal{T}}\right)_{\mathcal{R}}$ entails $M_{\mathcal{T}} \vDash^{\prime} E_{\mathcal{T}}$ by hypothesis, hence $M \models E$ by the satisfaction invariant. 
(ii) $\left(M_{\mathcal{T}}\right)_{\mathcal{R}} \subseteq \operatorname{Mod}(\operatorname{Th}(M))$ entails the implication $M \models E_{\mathcal{R}}^{\prime} \Rightarrow\left(M_{\mathcal{T}}\right)_{\mathcal{R}} \vDash E^{\prime}{ }_{\mathcal{R}}$, whence the validity of condition (i) under condition (ii) follows from the satisfaction invariant.

The conditions in the previous proposition also ensure full adequacy of the inverse transformation, as shown by the following.

Proposition 20. If $\mathcal{R}: \mathcal{I}^{\prime} \rightarrow \mathcal{I}$ is an inverse of $\mathcal{T}: \mathcal{I} \rightarrow \mathcal{I}^{\prime}$ such that $\forall \Sigma \in \operatorname{Sig}, \forall \mathrm{M} \in \operatorname{Mod}(\Sigma): M \in\left(\mathbf{M}_{\mathcal{T}}\right)_{\mathcal{R}}$, then

(i) $\mathcal{R}$ is fully adequate

(ii) $\mathcal{R} \circ \mathcal{T}$ is fully adequate

Proof.

(i) Let $\mathbf{M} \vDash\left(E_{j}^{\prime}\right)_{\mathcal{R}} \forall j \in J$. Since $M \in\left(M_{\tau}\right)_{\mathcal{R}}$ by hypothesis, $\exists M^{\prime} \in M_{\mathcal{T}}: M \in\left(M^{\prime}\right)_{\mathcal{R}}$. Furthermore, $M \models\left(E_{j}^{\prime}\right)_{\mathcal{R}} \Rightarrow M_{\mathcal{T}} \vDash^{\prime} E_{j}^{\prime}$ by Proposition $18(\mathrm{~d})$ and (a), therefore $\mathbf{M}^{\prime} \vDash \vDash^{\prime} \mathbf{E}_{j}^{\prime}$ for all $j \in J$.

(ii) Let $\mathrm{M} \models\left(\mathrm{E}_{j \tau}\right)_{\mathcal{R}}$ for all $j \in J$. Then $\mathrm{M}_{\mathcal{T}} \models^{\prime}\left(\mathrm{E}_{j}\right)_{\mathcal{T}}$ for all $j \in J$ according to Proposition $18(\mathrm{~d})$ and (a), hence $\mathrm{M} \vDash \mathrm{E}_{j}$ for all $j \in J$ by the satisfaction invariant, and $\mathrm{M} \in\left(\mathrm{M}_{\mathcal{T}}\right)_{\mathcal{R}}$ by hypothesis.

\section{Compactness}

In this Section we show the relevance of the basic framework outlined so far to the model-theoretic concept of compactness. In particular, we shall show the 'contravariant' (in the sense explained in Sect. 3) preservation of (two notions of) compactness by 'suitable' pre-institution transformations. The pre-institution transformation is to be 'suitable' in the sense that it.may have to fulfil certain requirements, such as (full) adequacy, depending on the property under consideration.

Definition 21. (Compactness)

Let $\mathcal{I}=($ Sig, Sen, Mod, $\models)$ be a pre-institution, with Pre $=\wp \circ$ Sen $:$ Sig $\rightarrow$ SET.

(i) $\mathcal{I}$ is compact iff $\forall \Sigma \in \operatorname{Sig}, \forall E \in \operatorname{Pre}(\Sigma): \mathrm{E}$ is satisfiable $\Leftrightarrow \mathrm{E}$ is finitely satisfiable.

(ii) $\mathcal{I}$ is consequence-compact iff $\forall \Sigma \in \operatorname{Sig}, \forall E \in \operatorname{Pre}(\Sigma), \forall \varphi \in \operatorname{Sen}(\Sigma)$ :

$\mathrm{E} \vDash \varphi \Rightarrow \exists \mathrm{F} \subseteq \mathrm{E}: \mathrm{F}$ finite $\wedge \mathrm{F} \vDash \varphi$.

The two notions of compactness are equivalent for pre-institutions that are closed under negation (see [11], p. 196, Lemma 2.1), where $\mathcal{I}$ is closed under negation whenever $\forall \Sigma \in \operatorname{Sig}, \forall \varphi \in \operatorname{Sen}(\Sigma), \exists \psi \in \operatorname{Sen}(\Sigma), \forall M \in \operatorname{Mod}(\Sigma)$ : $M \models \varphi$ iff not $M \models \psi$.

Since finiteness of (sub-)presentations plays an essential rôle in both notions of compactness, one may expect that 'suitable' pre-institution transformations for such notions ought to preserve that finiteness somehow. The basic, most intuitive idea is that every sentence should be transformed into a finite set of sentences. This idea is affected by too much of 'syntax', though, in the following sense.

If one accepts the abstract model-theoretic purpose proposed in [16], that is "to get away from the syntactic aspects of logic completely and to study classes of structures more in the spirit of universal algebra" then two softenings of the basic idea are in place. First, 'finiteness' of the transform $\varphi_{\mathcal{T}}$ of any sentence $\varphi$ should be 
measured excluding the 'tautological' part of $\varphi_{\mathcal{T}}$ ('tautological' relatively to $\mathcal{T}$, in a sense made precise below), since model classes are insensitive to tautologies. Second, and more generally in fact, 'finiteness' of sentence transformation should only be 'up to logical equivalence' in the target pre-institution, since logically equivalent sentences specify identical model classes.

The following definition tells, for a given pre-institution transformation, which sentences of the target pre-institution are 'viewed as tautologies' in the source preinstitution; we are thus considering a sort of 'stretching' of the classical notion of tautology along the transformation arrow. The subsequent definition, then, formalizes the two 'soft' forms of the property we are looking for, according to the rationale given above. The second, softer form is just what we need to prove the relevant part of the main theorem, which concludes the present analysis of compactness.

Definition 22. Let $\mathcal{T}: \mathcal{I} \rightarrow \mathcal{I}^{\prime}$ be a pre-institution transformation, with $\mathcal{I}, \mathcal{I}^{\prime}$ as in Definition 4. Then, for every $\Sigma \in$ Sig :

(i) a sentence $\psi \in \operatorname{Sen}\left(\Sigma_{\mathcal{T}}\right)$ is a $\mathcal{T}$-tautology iff $\forall \mathbf{M} \in \operatorname{Mod}(\Sigma), \forall \mathbf{M}^{\prime} \in \mathbf{M}_{\mathcal{T}}: \mathbf{M}^{\prime} \vDash \models^{\prime} \psi$,

(ii) $\operatorname{Taut}_{\mathcal{T}}(\Sigma)$ is the set of $\mathcal{T}$-tautologies in $\operatorname{Sen}\left(\Sigma_{\mathcal{T}}\right)$.

Clearly, every $\Sigma_{\mathcal{T}}$-tautology (in the classical sense) is in $\operatorname{Taut}_{\mathcal{T}}(\Sigma)$. This may contain more sentences, however, e.g. if $\emptyset_{\Sigma}$ is the 'empty' $\Sigma$-presentation, then clearly $\left(\emptyset_{\Sigma}\right)_{\mathcal{T}} \subseteq \operatorname{Taut}_{\mathcal{T}}(\Sigma)$, and the sentences in $\left(\emptyset_{\Sigma}\right)_{\mathcal{T}}$ need not be $\Sigma_{\mathcal{T}}$-tautologies.

Definition 23. Let $\mathcal{T}: \mathcal{I} \rightarrow \mathcal{I}^{\prime}$ be a pre-institution transformation, with $\mathcal{I}, \mathcal{I}^{\prime}$ as in Definition 4 .

(i) $T$ is fnitary iff $\forall \Sigma \in \operatorname{Sig}, \forall \varphi \in \operatorname{Sen}(\Sigma): \varphi_{T}-\operatorname{Taut}_{T}(\Sigma)$ finite.

(ii) $\mathcal{T}$ is quasi-finitary iff $\forall \Sigma \in \operatorname{Sig}, \forall \varphi \in \operatorname{Sen}(\Sigma):\left(\varphi_{\mathcal{T}}-\operatorname{Taut}_{\mathcal{T}}(\Sigma)\right) / \equiv \mathcal{I}^{\prime}$ finite.

The difference between finitarity and quasi-finitarity is illustrated by the transformation in Example 12, which is quasi-finitary but not necessarily finitary.

Theorem 24. (Compactness) Let $\mathcal{T}: \mathcal{I} \rightarrow \mathcal{I}^{\prime}$ be a fully adequate pre-institution transformation, with $\mathcal{I}, \mathcal{I}^{\prime}$ as in Definition 4.

(i) If $\mathcal{I}^{\prime}$ is compact, then $\mathcal{I}$ is compact.

(ii) If $\mathcal{I}^{\prime}$ is consequence-compact and $\mathcal{T}$ is quasi-finitary, then $\mathcal{I}$ is consequencecompact.

Proof.

(i) One direction of the compactness condition is trivial. Let then $\mathrm{E}$ be finitely satisfiable. If we can show that $\bigcup_{\psi \in E} \psi_{\mathcal{T}}$ is finitely satisfiable as well, then $\bigcup_{\psi \in E^{\prime}} \psi_{\mathcal{T}}$ is satisfiable by the hypothesis of compactness of $\mathcal{I}^{\prime}$, viz. there is a model $\mathbf{M}^{\prime}$ in $\mathcal{I}^{\prime}$ such that $\mathbf{M}^{\prime} \vDash \models^{\prime} \bigcup_{\psi \in E} \psi_{\mathcal{T}}$. Since $\mathcal{T}$ is fully adequate, $\mathbf{M}^{\prime} \in \mathrm{M}_{\mathcal{T}}$ and $\mathrm{M} \vDash \mathrm{E}$ for some model $\mathrm{M}$ in $\mathcal{I}$.

The finite satisfiability of $\bigcup_{\psi \in E} \psi_{T}$ is shown as follows.

Let $\mathrm{G}$ be any finite subset of $\bigcup_{\psi \in E} \psi_{\mathcal{T}}$.

For each $\chi \in G$ pick a $\psi_{\chi} \in \mathrm{E}$ such that $\chi \in\left(\psi_{\chi}\right) \tau$.

So $\mathbf{F}=\left\{\psi_{\chi} \mid \chi \in \mathrm{G}\right\}$ is a finite subset of $\mathbf{E}$, thus satisfiable by hypothesis.

There exists then a model $\mathbf{M}$ in $\mathcal{I}$ such that $\mathrm{M} \vDash \mathrm{F}$, thus $\forall \chi \in \mathrm{G}: \mathrm{M} \vDash \psi_{\chi}$, whence $\forall \chi \in G: M_{\mathcal{T}} F^{\prime}\left(\psi_{\chi}\right)_{\mathcal{T}}$ by the satisfaction invariant. Since $\forall \chi \in G: \chi \in\left(\psi_{\chi}\right)_{\mathcal{T}}$, we must conclude that $M_{\mathcal{T}} \vDash^{\prime} \mathrm{G}$, thus $\bigcup_{\psi \in E} \psi_{\mathcal{T}}$ is finitely satisfiable. 
(ii) Let $\mathrm{E} \vDash \varphi$ in $\mathcal{I}$, with $\varphi \in \operatorname{Sen}(\Sigma)$ and $\mathrm{E} \in \operatorname{Pre}(\Sigma)$ for some $\Sigma \in \operatorname{Sig}$. Then $\mathrm{E}_{\mathcal{T}} \vDash \varphi_{\mathcal{T}}$ by adequacy of $\mathcal{T}$ and Proposition 16(ii), thus $\bigcup_{\psi \in E} \psi_{\mathcal{T}} \vDash \varphi_{\mathcal{T}}$ by full adequacy of $\mathcal{T}$ and Proposition 16(iv), that is $\bigcup_{\psi \in E} \psi_{T} \vDash \xi$ for all $\xi \in \varphi_{T}$. Consequencecompactness of $\mathcal{I}^{\prime}$ implies that for each $\xi \in \varphi_{\mathcal{T}}$ there exists a finite $\mathrm{G}_{\xi} \subseteq \bigcup_{\psi \in E} \psi_{\mathcal{T}}$ such that $\mathrm{G}_{\xi} \vDash \xi$.

Now, for each $\chi \in \mathrm{G}_{\xi}$ pick a $\psi_{\chi} \in \mathrm{E}$ such that $\chi \in\left(\psi_{\chi}\right)_{\mathcal{T}}$.

Let $\mathbf{F}_{\xi}=\left\{\psi_{\chi} \mid \chi \in \mathrm{G}_{\xi}\right\} \subseteq \mathrm{E}$. Moreover, let $\widehat{\varphi \mathcal{T}}$ be a set of representatives for the equivalence classes in the quotient $\left(\varphi_{\mathcal{T}}-\right.$ Taut $\left._{\mathcal{T}}(\Sigma)\right) / \equiv_{\mathcal{I}^{\prime}}$, with $\widehat{\varphi_{\mathcal{T}}}$ consisting of exactly one representative per equivalence class. Define then $F=\bigcup_{\xi \in \widehat{\varphi_{T}}} F_{\xi}$.

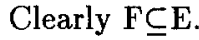

$F$ is finite since $\mathrm{G}_{\xi}$ finite implies $\mathrm{F}_{\xi}$ finite and $T$ quasi-finitary implies that $\left(\varphi_{\mathcal{T}}-\right.$ Taut $\left._{\mathcal{T}}(\Sigma)\right) / \equiv_{\mathcal{I}}$, is finite, hence $\widehat{\varphi_{\mathcal{T}}}$ finite. $\mathrm{G}_{\xi} \vDash \xi$ and $\mathrm{G}_{\xi} \subseteq \bigcup_{\chi \in G_{\xi}}\left(\psi_{\chi}\right)_{\mathcal{T}}$ together entail $\bigcup_{\chi \in G_{\xi}}\left(\psi_{\chi}\right)_{\tau} \vDash \xi$, which, by the definition of $\mathbf{F}_{\xi}$, is equal to $\bigcup_{\psi \in F \xi} \psi_{\mathcal{T}} \vDash \xi$. This, together with the definition of $\mathrm{F}$ thus yield $\bigcup_{\psi \in F} \psi_{\mathcal{T}} \vDash \widehat{\varphi_{\mathcal{T}}}$, therefore $\bigcup_{\psi \in F} \psi_{\mathcal{T}} \vDash \varphi_{\mathcal{T}}$-Taut Tau $_{\mathcal{T}}(\Sigma)$ since consequence in $\mathcal{I}^{\prime}$ is preserved under $\mathcal{I}^{\prime}$-equivalence. Now, $\bigcup_{\psi \in F} \psi_{\mathcal{T}} \vDash \operatorname{Taut}_{\mathcal{T}}(\Sigma)$ if for some $\psi \in \mathrm{F} \psi_{\mathcal{T}} \vDash \operatorname{Taut}_{\mathcal{T}}(\Sigma)$, which easily follows from adequacy of $\mathcal{T}$ and Proposition 16(ii) together with Definition 22. Thus $\bigcup_{\psi \in F} \psi_{\mathcal{T}} \vDash \varphi_{\mathcal{T}}$, whence $\mathrm{F}_{\mathcal{T}} \vDash \varphi_{\mathcal{T}}$ by adequacy of $\mathcal{T}$ and Proposition 16(iii), and thus finally $\mathrm{F} \models \varphi$ by Proposition 16(i).

\section{Application Examples}

In [18], among others, translations of order-sorted conditional equational logic [14] and of conditional equational logics for partial algebras $[1,2,7,8,22]$ into equational type logic [17] were made available, and were shown sound and complete with respect to deduction, as well as with respect to translation of signature declarations by equational type axioms.

In this Section, we slightly extend each of the aforementioned translations, to obtain pre-institution transformations which are easily shown to be fully adequate. The completeness results are then immediate consequence of Proposition 16 (soundness being ensured by the satisfaction invariant).

\subsection{Order-Sorted Algebra}

We recall the translation defined in [18] for order-sorted conditional equational logic, that consists of the following:

1. If $\Sigma=\left\langle\mathrm{S}, \leq, \Sigma_{\text {op }}\right\rangle$ is an order-sorted signature, its translation $\Sigma_{\mathcal{T}}$ is the unsorted signature that has as 0 -ary operators the nullary operators of $\Sigma$ and the sorts in $S$, and as $k$-ary operators the operators of $\Sigma_{o p}$ with $k$ arguments, for $k>0$.

2. If $\mathrm{E}$ is a set of order-sorted $\Sigma$-sentences, thus a set of conditional equations over an order-sorted signature $\Sigma$ as in 1 above and a system of variables $V$ on $\Sigma$, let $V_{\mathcal{T}}=|\mathrm{V}| \cup \mathrm{Y}$, where $|\mathrm{V}|$ is the underlying set of variables in $\mathrm{V}$ and $\mathrm{Y}$ $=\left\{\mathrm{y}, \mathrm{y}_{1}, \ldots, \mathrm{y}_{n}, \ldots\right\}$ is a countable set of new variables (viz. $|\mathrm{V}| \cap \mathrm{Y}=\emptyset$ ). The translation $\mathbf{E}_{\mathcal{T}}$ is the smallest set of $\Sigma_{\mathcal{T}}$-sentences of equational type logic that contains: 
(a) for each operator $\omega: s_{1}, \ldots, s_{n} \rightarrow \mathrm{s}$ in $\Sigma_{o p}$, the $\Sigma_{\mathcal{T}}$-sentence

$(\omega)_{\mathcal{T}} \stackrel{\text { def }}{=} \mathrm{y}_{1}: \mathrm{s}_{1}, \ldots, \mathrm{y}_{n}: \mathrm{s}_{n} \rightarrow \omega\left(\mathrm{y}_{1}, \ldots, \mathrm{y}_{n}\right): \mathrm{s}$

(b) for each sort inclusion $s \leq s^{\prime}$ in $\Sigma$, the $\Sigma_{\mathcal{T}}$-sentence $\left(\mathrm{s} \leq \mathrm{s}^{\prime}\right) \tau \stackrel{\text { def }}{=} \mathrm{y}: \mathrm{s} \rightarrow \mathrm{y}: \mathrm{s}^{\prime}$

(c) for each $\Sigma$-sentence $\varphi=\forall X . e_{1}, \ldots, e_{n} \rightarrow$ in $\mathrm{E}$, the $\Sigma_{\mathcal{T}^{-}}$-sentence $\varphi_{T} \stackrel{\text { def }}{=} \mathrm{x}_{1}: \mathrm{s}_{1}, \ldots, \mathrm{x}_{m}: \mathrm{s}_{m}, \mathrm{e}_{1}, \ldots, \mathrm{e}_{n} \rightarrow \mathrm{e}$ with $\mathrm{x}_{1}, \ldots, \mathrm{x}_{m}$ the variables of $\varphi$, thus in $\mathrm{X}, \mathrm{s}_{1}, \ldots, \mathrm{s}_{m}$ their respective sorts therein.

3. If $\mathbf{M}$ is an order-sorted $\Sigma$-algebra, its translation $\mathbf{M}_{\mathcal{T}}$ is the closure under isomorphism of the set of $\Sigma_{T}$ :-algebras $\mathrm{M}^{\prime}$ that meet the following requirements: carrier: $\left|\mathbf{M}^{\prime}\right| \supseteq \bigcup_{\mathbf{s} \in \mathbf{S}} \mathbf{M}_{\mathbf{S}}$ typing: for every $\mathrm{s} \in \mathrm{S}, \mathrm{a}:{ }_{M^{\prime}} \mathrm{s}^{M^{\prime}} \Leftrightarrow \mathrm{a} \in \mathrm{M}_{\mathrm{S}}$ operations: for every $\omega: \mathrm{s}_{1}, \ldots, \mathrm{s}_{n} \rightarrow \mathrm{s}$ in $\Sigma_{o p}$,

$$
\omega^{M^{\prime}}\left(\mathrm{a}_{1}, \ldots, \mathrm{a}_{n}\right)=\omega^{M}\left(\mathrm{a}_{1}, \ldots, \mathrm{a}_{n}\right) \text { if } \mathrm{a}_{1}:_{M^{\prime}} \mathrm{s}_{1}^{M^{\prime}}, \ldots, \mathrm{a}_{n}:_{M^{\prime}} \mathrm{s}_{n}^{M^{\prime}} \text {. }
$$

To obtain a pre-institution transformation from the above translation, one must define the translation of order-sorted signature morphisms, check the naturality of the presentation as well as model transformations, and check the validity of the satisfaction invariant.

The first item in this task is easy: if $\tau: \Sigma_{1} \rightarrow \Sigma_{2}$ is an order-sorted signature morphism, define $\tau_{\mathcal{T}} \omega=\tau \omega$ for all $\omega$ in $\Sigma_{1 \tau}$. This yields naturality of the presentation transformation, as it is easy to check.

The remaining items preliminarly require the choice of the appropriate notion of model reduction in equational type logic. Order-sorted (as well as many-sorted) reduction may forget not only operations but also carriers; precisely, it forgets those carriers that have no designation in the image of the signature morphism. In [17] we argued, on intuitive grounds, that algebraic $\langle 1: 0\rangle$-reduction in equational type logic was an appropriate reflection of this feature. To see this, we first recall a few notions relating to algebraic reduction in equational type logic.

First, relatively to a signature morphism ${ }^{8} \tau: \Omega \rightarrow \Omega^{\prime}$, the standard reduct of an $\Omega^{\prime}$-signed type algebra (or $\Omega^{\prime}$ :-algebra) $\mathcal{A}^{\prime}$ is the $\Omega$ :-algebra $\mathcal{A}$ that has the same carrier and typing, and operators' interpretation $\omega^{\mathcal{A}} \stackrel{\text { def }}{=}(\tau \omega)^{\mathcal{A}^{\prime}}$ for all $\omega \in \Omega$. Thus, like with universal algebras, no elements are forgotten by standard reduction, but only operations. Then, relatively to a signature morphism $\tau: \Omega \rightarrow \Omega^{\prime}$, the $\langle 0: 0\rangle$-reduct (actually a subreduct) of an $\Omega^{\prime}$ :-algebra is the least $\Omega$-subalgebra of the standard reduct of that type algebra. Finally, relatively to a signature morphism as above, a $\langle 1: 0\rangle$-reduct of a type algebra is the least subalgebra of the standard reduct that contains every element that is typed by some element of the $\langle 0: 0\rangle$-reduct (which is thus a subalgebra of the $\langle 1: 0\rangle$-reduct as well).

Now, the present framework enables us to confirm our aforementioned expectation on formal grounds, since the model transformation as defined above enjoys naturality under $\langle 1: 0\rangle$-reduction only.

\footnotetext{
8 i.e., an arity-preserving map on the operation symbols
} 
In passim, we note that equational type logic with $\langle 1: 0\rangle$-reduction is rps (see Definition 2), but not eps, ${ }^{9}$ hence not ps, whilst the order-sorted logic of our concern, as well as many-sorted conditional equational logic, do enjoy the ps property. Proposition 15 then entails that the transformation above has no inverse; more generally, it entails that a search for a pre-institution transformation in the converse direction to that here exemplified makes sense for equational type logic with standard reduction only.

Validity of the satisfaction invariant is the content of Proposition 3.5 in [18], whose Proposition 3.6 shows adequacy of the transformation above. Finitarity of the transformation is evident. Note that adequacy and full adequacy coincide in this example because of the elementwise definition of the presentation transformationwhich may be expected to be the case in many practical situations.

\subsection{Partial Algebra}

We recall that unsorted partial algebras differ from total ones in that the carrier of a partial algebra need not be closed w.r.t. application of the operations of the algebra. As a matter of notation, $\omega^{\mathcal{A}} \downarrow\left\langle a_{1}, \ldots, a_{n}\right\rangle$ means that the application of the $n$-ary operation $\omega^{\mathcal{A}}$ to argument $\left\langle a_{1}, \ldots, a_{n}\right\rangle \in|\mathcal{A}|^{n}$ is defined in $\mathcal{A}$. A strictness requirement applies to definedness of terms representing such applications, saying that if the interpretation of a ground term is defined, then so must be the interpretation of every subterm of that term.

We further recall that the conditional equational logics of unsorted partial algebras considered in [18] may have weak as well as strong conditional equations as sentences. These respectively follow two variants of the common form $e_{1}, \ldots, e_{n} \rightarrow e$, with $e_{1}, \ldots, e_{n}$ weak equations in both cases, and $e$ a weak, resp. strong equation. The two variants differ in the conclusion of the conditional, thus. This form can cater for definedness predicates, since $D(t)$ is equivalent to the weak equation $t \stackrel{w}{\equiv} t$, but not for the partial conditional equations considered in [3], which admit strong equations in the premiss; that logic does not always have initial models, hence its faithful translation in equational type logic is not possible.

The translation in [18] of the aforementioned logics into equational type logic consists of the following:

1. If $\Omega$ is an unsorted partial algebra signature, its translation $\Omega_{\mathcal{T}}$ is the extension of $\Omega$ with the nullary operator $T$ (assuming $T \notin \Omega$ ).

2. If $\mathrm{E}$ is an unsorted conditional equational partial algebra presentation, viz. a set of weak or strong conditional equations over an unsorted signature $\Omega$ and a set of variables $\mathrm{V}$, let $\Omega_{\mathcal{T}}$ be the signature as in 1 above, and $V_{T}=V \cup Y$, where $\mathrm{Y}=\left\{\mathrm{y}_{1}, \ldots, \mathrm{y}_{n}, \ldots\right\}$ is a countable set of new variables (viz. $\mathrm{V} \cap \mathrm{Y}=\emptyset$ ). The translation $\mathrm{E}_{\mathcal{T}}$ is then the smallest set of $\Omega_{\mathcal{T}}$-sentences of equational type logic that contains:

\footnotetext{
${ }^{9}$ Failure of the eps property in equational type logic with $\langle 1: 0\rangle$-reduction is shown by the following counterexample. Consider a two-element type algebra, on a signature consisting of one nullary operator only, and with the typing relation empty. Relatively to the identity signature morphism, the $\langle 1: 0\rangle$-reduct coincides with the $\langle 0: 0\rangle$-reduct in this case, hence it satisfies the equation $x=x$, which is not valid in the original type algebra.
} 
(a) for each $n$-ary operator $\boldsymbol{\omega}$ in $\Omega$, the following $n \Omega_{\mathcal{T}}$-sentences:

$\omega\left(\mathrm{y}_{1}, \ldots, \mathrm{y}_{n}\right): \mathrm{T} \rightarrow \mathrm{y}_{1}: \mathrm{T}, \ldots, \omega\left(\mathrm{y}_{1}, \ldots, \mathrm{y}_{n}\right): \mathrm{T} \rightarrow \mathrm{y}_{n}: T$

(b) for each weak conditional equation $\mathrm{t}_{1} \stackrel{w}{\equiv} \mathrm{u}_{1}, \ldots, \mathrm{t}_{n} \stackrel{w}{\equiv} \mathrm{u}_{n} \rightarrow \mathrm{t} \stackrel{w}{\equiv} \mathrm{u}$ the following two $\Omega_{\mathcal{T} \text {-sentences: }}$

$\mathrm{t}_{1}: \mathrm{T}, \ldots, \mathrm{t}_{n}: \mathrm{T}, \mathrm{t}_{1} \equiv \mathrm{u}_{1}, \ldots, \mathrm{t}_{n} \equiv \mathrm{u}_{n} \rightarrow \mathrm{t}: \mathrm{T}$

$t_{1}: T, \ldots, t_{n}: T, t_{1} \equiv u_{1}, \ldots, t_{n} \equiv u_{n} \rightarrow t \equiv u$

(c) for each strong conditional equation $\mathrm{t}_{1} \stackrel{w}{=} \mathrm{u}_{1}, \ldots, \mathrm{t}_{n} \stackrel{w}{=} \mathrm{u}_{n} \rightarrow \mathrm{t} \stackrel{s}{\equiv} \mathbf{u}$ the following two $\Omega_{\mathcal{T}}$-sentences:

$\mathrm{t}_{1}: \mathrm{T}, \ldots, \mathrm{t}_{n}: \mathrm{T}, \mathrm{t}_{1} \equiv \mathrm{u}_{1}, \ldots, \mathrm{t}_{n} \equiv \mathrm{u}_{n}, \mathrm{t}: \mathrm{T} \rightarrow \mathrm{t} \equiv \mathrm{u}$

$t_{1}: T, \ldots, t_{n}: T, t_{1} \equiv u_{1}, \ldots, t_{n} \equiv u_{n}, u: T \rightarrow t \equiv u$

3 . If $\mathrm{M}$ is an unsorted partial $\Omega$-algebra, its translation $\mathbf{M}_{\mathcal{T}}$ is the closure under isomorphism of the set of $\Omega_{\mathcal{T}}$ :-algebras $\mathrm{M}^{\prime}$ that meet the following requirements: carrier: $\left|\mathbf{M}^{\prime}\right| \supseteq|\mathbf{M}|$

typing: $\mathrm{a}:{ }_{M^{\prime}} \mathrm{T}^{M^{\prime}}$ iff $\mathrm{a} \in|\mathrm{M}|$

operations: the constant $T^{M^{\prime}}$, and for each $n$-ary operator $\omega \in \Omega, \forall \mathrm{a}_{1}, \ldots, \mathrm{a}_{n} \in\left|\mathbf{M}^{\prime}\right|$ :

$$
\begin{aligned}
& \text { (i) }\left(\mathrm{a}_{1}:_{M^{\prime}} \mathrm{T}^{M^{\prime}} \wedge \ldots \wedge \mathrm{a}_{n}:_{M^{\prime}} \mathrm{T}^{M^{\prime}} \wedge \omega^{M} \downarrow\left\langle\mathrm{a}_{1}, \ldots, \mathrm{a}_{n}\right\rangle\right) \\
& \Rightarrow \omega^{M^{\prime}}\left(\mathrm{a}_{1}, \ldots, \mathrm{a}_{n}\right)=\omega^{M}\left(\mathrm{a}_{1}, \ldots, \mathrm{a}_{n}\right) \\
& \text { (ii) } \omega^{M^{\prime}}\left(\mathrm{a}_{1}, \ldots, \mathrm{a}_{n}\right):_{M^{\prime}} \mathrm{T}^{M^{\prime}} \\
& \Rightarrow\left(a_{1}:{ }_{M^{\prime}} T^{M^{\prime}} \wedge \ldots \wedge a_{n}:_{M^{\prime}} T^{M^{\prime}} \wedge \omega^{M} \downarrow\left\langle a_{1}, \ldots, a_{n}\right\rangle\right)
\end{aligned}
$$

To obtain a pre-institution transformation from the above translation, we define the translation of signature morphisms as follows: if $\tau: \Omega_{1} \rightarrow \Omega_{2}$ is an unsorted signature morphism, then $\tau_{\tau}{ }^{\top}=T$ and $\tau_{\tau} \omega=\tau \omega$ for all remaining $\omega$ in $\Omega_{1 \tau}$. It is easy to check that this yields naturality of the presentation transformation, and naturality of the model transformations as well as validity of the satisfaction invariant, under the standard algebraic reduction in equational type logic (because of the unsortedness of the partial algebras of our concern, here).

Validity of the satisfaction invariant is the content of Proposition 4.6 in [18], whose Proposition 4.7 shows adequacy of the transformation above. Finitarity of the transformation is evident. Adequacy and full adequacy here coincide, again because of the elementwise definition of the presentation transformation.

We conclude by noting that, in this example, the possibility of translating a sentence by a set of sentences proves indeed useful; had one to work with pointwise sentence translation, a preliminary lifting to pre-institutions with sentences formed by (finite) sets of sentences would have been necessary, which would have made the translation quite indirect.

\section{Related Work}

This work shares with [4] the motivation for focussing on the morphisms of institution categories more than on their objects. Clearly, reason for this is the interest that institution-independent specification [24] and general logics [10,19] naturally find in computer science. It may be source of some surprise, thus, that so far no general agreement has been reached on the most convenient notion of institution 
morphism. A careful analysis and comparison of several, quite distinct such notions can be found in [4]. To that, we wish to add the following, necessarily quick and preliminary considerations.

In comparison with the notions of institution morphism proposed in $[13,19,4]$, our notion is the only one where the three arrows (respectively relating to signatures, sentences, models) are all covariant. Of those three notions, the one which seems closest to ours is that of basic simulation [4], which essentially differs from ours in two respects: 1) it sends sentences to sentences, whereas ours sends presentations to presentations, and 2) model transformation is contravariant, but by a surjective, partial natural transformation. The latter could thus be turned into a covariant, total natural transformation, sending models to sets of models, like in our case. Of course, the model sets ought to be disjoint; this condition, arising from well-definedness of the model transformation as defined for basic simulations, closely corresponds to the sufficient condition for full adequacy given in Lemma 8 above-modulo some equivalence of models in the source institution, however.

Although more interested in the morphisms than in the objects, the less restrictive definition of the objects in our framework also contributes to widening the applicability of abstract model-theoretic tools in algebraic specification. Most of the specification frameworks studied so far, fit the institutions scheme, yet not all of them do. Cases where (at least the eps half of) the satisfaction condition is known to fail include equational type logic with non-standard reduction (as exemplified in Sect. 5) and, most notably, behavioural semantics [6, 20, 21]. These frameworks fit the pre-institutions scheme, as well as the drastically general scheme proposed in [12] - where hardly anything of the abstract model-theoretic approach underlying the theory of institutions can be recognized, though.

\section{Conclusions}

We revisit the motivation for the present work in the light of the results obtained in Sects. 3 and 4, and draw a couple of conclusions from the application examples worked out in Sect. 5. We finally mention a few topics of future work.

We motivated our present study by arguing the interest of investigating which properties of institutions do actually depend on the satisfaction condition and/or on the categorial structure on models, and which do not. Now, the results in Sects. 3 and 4 of the present paper offer a first blend of properties which do not depend on either feature of institutions, thus holding for the larger class of pre-institutions.

The blend is further, significantly extended in [23]; there, however, we also obtain a result where a slightly weaker form of the ps property is made use of. This is the renaming property [10], which is just the ps property but limited to signature isomorphisms only. The result which depends on this property is a straightforward generalization of the Hanf theorem to pre-institutions with cardinal numbers. The Hanf theorem, giving 'smallness' conditions that ensure existence of Hanf numbers (and a similar theorem on the existence of Löwenheim numbers) is quite fundamental in abstract model theory, and certainly does make a strong case for the renaming property. This does not invalidate the approach followed in the present paper, however, where we have favoured the analysis of the effect of properties a posteriori to their a priori requirement. 
A not dissimilar viewpoint motivates the absence of categorial structure on models in pre-institutions. Morphisms between models 'exist in nature'; no account of them is taken in the notion of pre-institution for the simple reason that they play no rôle in establishing merely logical results like those presented in this paper, as well as those in [23]-and potentially many others, we guess. But model morphisms, and (properties of) their transformations especially, certainly have a rôle to play for investigations of greater mathematical depth, such as algebraic ones. There, the categorially richer structure of institutions is surely well-motivated, in fact necessary. We expect the work on pre-institutions to prove still useful in that wider context, for instance to gain more modularity in the application of the theory of institutionswhich fact seems interesting from a 'theory engineering' viewpoint.

So far about theory and its motivations. Concerning applications, two facts seem apparent from the analysis worked out in Sect. 5. On the one hand, the recasting of the translations of our concern into the formal structure of a pre-institution transformation slightly increases the construction work (translation of signature morphisms) and slightly decreases the proof work (completeness with respect to deduction). On the other hand, however, the proof work is made easier somewhat, in that general requirements and facts which ensure soundness and other desirable properties are known in advance. In other words, a proof structure is available in the general framework, that the specific transformation design has to 'fill in' with appropriate details.

Finally, topics for future work that attract our current interest include: the generalization of abstract model theory towards model independence (what could be properly understood as 'soft model theory', where 'soft' applies to 'model' no less than to 'theory'); the investigation of initiality and freeness in this framework (for which $[25,26]$ offer a solid starting point); the study of whether and how could the present framework be extended to cater for proof calculi [19]; and, last but not least, the investigation of applications, whereby further insight is to be expected.

\section{References}

1. Andréka, H., Burmeister, P., Németi, I.: Quasivarieties of Partial Algebras - A Unifying Approach towards a Two-Valued Model Theory for Partial Algebras. Studia Sci. Math. Hungar. 16 (1981) 325-372

2. Andréka, H., Németi, I.: Generalization of Variety and Quasivariety Concepts to Partial Algebras through Category Theory. Dissertationes Mathematicæ (Rozprawy Mathematyczne) 204 (Warsaw, 1983)

3. Astesiano E., Cerioli, M.: On the Existence of Initial Models for Partial (Higher-Order) Conditional Specifications, In: Diaz, J., Orejas, F. (Eds.) TAPSOFT'89, LNCS 351 (Springer-Verlag, Berlin, 1989) 74-88

4. Astesiano E., Cerioli, M.: Commuting between Institutions via Simulation. University of Genova, Formal Methods Group, Technical Report n. 2 (1990)

5. Barwise, K. J.: Axioms for Abstract Model Theory. Ann. Math. Logic 7 (1974) 221-265

6. Bernot, G., Bidoit, M.: Proving the Correctness of Algebraically Specified Software: Modularity and Observability Issues. Proc. 2nd Int. Conf. Algebraic Methodology And Software Technology, Iowa City, (lowa, USA, May 22-25, 1991) 139-161

7. Broy, M., Wirsing, M.: Partial Abstract Types. Acta Informatica, 18, 3 (1982) 47-64 
8. Burmeister, P.: A Model-Theoretic Oriented Approach to Partial Algebras. Mathematical Research, Volume 31, (Akademie-Verlag, Berlin, 1986)

9. Chang, C.C., Keisler, H.J.: Model Theory. Third Edition (North-Holland, Amsterdam, 1990)

10. Ebbinghaus, H.-D.: Extended logics: the general framework. In: Barwise, J., Feferman, S. (Eds.) Model-Theoretic Logics (Springer-Verlag, Berlin, 1985) 25-76

11. Ebbinghaus, H.-D., Flum, J., Thomas, W.: Mathematical Logic. (Springer-Verlag, New York, 1984)

12. Ehrig, H., Baldamus, M., Cornelius, F., Orejas, F.: Theory of Algebraic Module Specification Including Behavioural Semantics, Constraints and Aspects of Generalized Morphisms. Proc. 2nd Int. Conf. Algebraic Methodology And Software Technology, Iowa City, (Iowa, USA, May 22-25, 1991) 101-125

13. Goguen, J.A., Burstall, R.: Introducing Institutions. In: Clarke, E., Kozen, D. (Eds.), Logics of Programs, LNCS 164 (Springer-Verlag, Berlin, 1984) 221-256

14. Goguen, J.A., Meseguer, J.: Order-Sorted Algebra I: Equational Deduction for Multiple Inheritance, Overloading, Exceptions and Partial Operations. Report SRI-CSL-89-10, SRI International, Computer Science Laboratory (Menlo Park, California, July 1989)

15. Mac Lane, S.: Categories for the Working Mathematician. Graduate Texts in Mathematics, Volume 5 (Springer-Verlag, New-York, 1971)

16. Makowski, J.-A.: Compactness, Embeddings an Definability. In: Barwise, J., Feferman, S. (Eds.) Model-Theoretic Logics (Springer-Verlag, Berlin, 1985) 645-716

17. Manca, V., Salibra, A., Scollo, G.: Equational Type Logic. Theoret. Comput. Sci. 77 (1990) 131-159

18. Manca, V., Salibra, A., Scollo, G.: On the Expressiveness of Equational Type Logic. In: Rattray, C.M.I., Clark R.G. (Eds.) The Unified Computation Laboratory, Proc. IMA Conf., Stirling, Scotland, July 3-6, 1990; to appear (Oxford University Press, 1992)

19. Meseguer, J.: General Logics. In: Ebbinghaus, H.-D. et al. (Eds.) Logic Colloquium'87 (North-Holland, Amsterdam, 1989) 275-329

20. Nivela, M.P., Orejas, F.: Initial Behaviour Semantics for Algebraic Specifications. In: Sannella, D.T., Tarlecki, A. (Eds.) Recent Trends in Data Type Specification, LNCS 332 (Springer-Verlag, Berlin, 1988) 184-207

21. Orejas, F., Nivela, M.P., Ehrig, H.: Semantical Constructions for Categories of Behavioural Specifications. In: Ehrig, H., Herrlich, H., Kreowski, H.-J., Preuß, G. (Eds.) Categorical Methods in Computer Science - with Aspects from Topology, LNCS 393 (Springer-Verlag, Berlin, 1989) 220-243

22. Reichel, H.: Initial Computability, Algebraic Specifications, and Partial Algebras. The International Series of Monographs on Computer Science, Volume 2 (Oxford University Press, Oxford, 1987)

23. Salibra, A., Scollo, G.: Compactness and Löwenheim-Skolem Properties in PreInstitution Categories. Memoranda Informatica 92-27, TIOS 92/12, University of Twente, March 1992; submitted for publication.

24. Sannella, D.T., Tarlecki, A.: Specifications in an Arbitrary Institution. Information and Computation 76 (1988) 165-210

25. Tarlecki, A.: On the Existence of Free Models in Abstract Algebraic Institutions. Theoret. Comput. Sci. 37 (1985) 269-304

26. Tarlecki, A.: Quasi-Varieties in Abstract Algebraic Institutions, J. of Computer and System Sciences 33 (1986) 333-360 